\title{
ESTUDO COMPARATIVO DO USO DE EXTRATO DE PFAFFIA GLOMERATA E DO LASER DE BAIXA POTÊNCIA (HÉLIO-NEÔNIO) NA CICATRIZAÇÃO DE FERIDAS EM RATOS
}

\author{
Comparative study of the use of Pfaffia glomerata and the low power laser (Helium-Neon) in \\ the healing of the wounds in rats
}

\author{
Marileide Inácio da Silva CARNEIRO ${ }^{1}$, Jurandir Marcondes RIBAS FILHO', Osvaldo MALAFAIA ${ }^{1}$, \\ Carmen Australia Paredes Marcondes RIBAS', Cid Aimbiré M. SANTOS², \\ Tereza Cristina Santos CAVALCANTI ${ }^{1}$, Letícia Elizabeth Augustin CZECZKO ${ }^{1}$
}

Trabalho realizado no ${ }^{1}$ Instituto de Pesquisas Médicas - IPEM do Hospital Universitário Evangélico de Curitiba/Faculdade Evangélica do Paraná e 2Laboratório de Farmacognosia, Departamento de Farmácia, Universidade Federal do Paraná, Curitiba, PR, Brasil.

DESCRITORES - Cicatrização. Ratos. Fitoterapia. Laser. Estudo comparativo.
RESUMO - Racional - A Pfaffia glomerata é planta utilizada na medicina popular como tônico, antidiabético, para melhorar o processo de cicatrização, neutralizar distúrbios gástricos e antirreumático. Objetivos - Comparar a contração da ferida e a neovascularização com o uso da Pfaffia glomerata e do laser de baixa potência em dorso de ratos. Métodos - Foram utilizados 40 ratos da linhagem Wistar nos quais realizaram-se feridas cirúrgicas com punch de $3 \mathrm{~mm}$ de diâmetro na parte superior direita do dorso onde nenhuma substância foi aplicada e nesses mesmos animais foram também realizadas feridas cirúrgicas no lado esquerdo na região inferior, onde foi aplicado o extrato das raízes de Pfaffia glomerata (Spreng.) Pedersen, Amaranthaceae, obtendo-se desta maneira os grupos controle e planta nos mesmos animais. Em outros 40 animais, foi utilizado o laser de baixa potência. Nas datas programadas em 48 horas, uma, duas e três semanas foram tomadas as medidas da contração da ferida. Microscopicamente os resultados foram analisados utilizando-se imunoistoquímica como fator VIII para observar a densidade vascular. Resultados - Macroscopicamente não houve diferença estatisticamente significante com relação à contração da ferida entre os grupos planta e laser, obtendo ambos resultados superiores ao grupo controle. Dentro desta variável, o resultado com diferença estatisticamente significante ocorreu dentro do grupo laser na comparação dos subgrupos: 48 horas vs. uma semana $(p=0,008)$. A análise do Fator VIII mostrou significância estatística no subgrupo de uma semana da planta em relação ao laser $(p=0,09)$. Conclusões: Não houve diferença estatisticamente significante com relação à contração da ferida entre os grupos planta e laser, obtendo ambos resultados superiores ao grupo controle no final do estudo; contudo, microscopicamente, o grupo planta obteve valor superior ao grupo laser quanto à neovascularização em uma semana, mas após esse período, houve equilíbrio com os outros grupos.

\section{Correspondência:}

Marileide Inácio da Silva Carneiro

e-mail:mari_inacio70@hotmail.com

Fonte de financiamento: não há

Conflito de interesses: não há

Recebido para publicação: 19/03/2010

Aceito para publicação: 14/06/2010

HEADINGS- Surgery. Rats. Phytotherapy. Laser. Comparative study.
ABSTRACT - Background - Pfaffia glomerata is a plant used in folk medicine as tonic, antidiabetic, to improve the healing process, neutralize stomach upset and antirheumatic. Aim - To compare the wound contraction and the neovascularization with the use of plant extract of Pfaffia glomerata (Spreng.) Pedersen, Amaranthaceae, and low power laser in rats. Methods - It was used 40 Wistar rats in which surgical wounds with a punch of $3 \mathrm{~mm}$ in diameter at the top right of the back and no substance was applied at this site, and similar wounds in the lower left side had applicated extract of P. glomerata (Spreng.) Pedersen, Amaranthaceae, control groups and plants in the same animal. Another 40 animals had the low power laser application. On dates scheduled within 48 hours, one, two and three weeks measures were taken and wound contraction observed. Microscopic results were analyzed using immunohistochemistry with Factor VIII to observe the vascular density. Results - It was possible to observe macroscopically no statistic significant difference with respect to the contraction of the wound between the groups plant and laser, both getting better results than the control group. This variable showed a statistical significance with the laser group when was compared the subgroups 48 hours $X$ one week $(p=0,008)$. Statistical analysis of Factor VIII showed an statistic significance in subgroup one week of the plant with the laser $(p=0.09)$. Conclusions - There was no statistic significant difference between the plant and laser groups with respect to contraction of the wound. On microscopic analysis, the group plant earned more than the laser group with relation to neovascularization in subgroup one week, and after that occured a balance between them. 
INTRODUÇÃO

pele é responsável pela interface entre o
corpo e o ambiente e por isto é adaptada
para servir a muitas funções diferentes. Ela está constantemente relacionada com atividades biológicas e bioquímicas. É composta de duas camadas primárias. A externa, chamada de epiderme, constituise de tecido epitelial. A interna, denominada de derme, é formada de tecido conjuntivo. As duas estão unidas através da membrana basal. A epiderme possui interdigitações, que são projeções para o interior da derme, evitando desta maneira o descolamento das duas durante tensões externas.

A perda tecidual pode atingir a derme parcial ou total chegando ao tecido celular subcutâneo. Baseado nisto, foram definidos dois tipos de ferida: a de espessura parcial e a de espessura total ${ }^{7}$.

Quando a ferida cirúrgica é de espessura parcial, dá-se o nome de derme incompleta e a reparação faz-se pela re-epitelização dos anexos epiteliais ou epitélio derivado da pele adjacente não acometida. Como resultado final tem-se cicatriz praticamente imperceptível.

Quando as feridas são de espessura total, diz-se derme completa ou estendida ao tecido celular subcutâneo; há necessidade de formação de um novo tecido, o de granulação. A epitelização - base da cicatrização nas feridas de espessura parcial -, acontece apenas nas margens e a cicatriz é totalmente perceptível, muitas vezes pronunciada.

A cicatrização também depende de vários fatores locais e gerais, como: localização anatômica, tipo da pele, raça e técnica cirúrgica utilizada.

Diferentes classificações são utilizadas para facilitar o entendimento de um processo totalmente dinâmico e com fases tão interdependentes. A forma mais completa divide o processo de cicatrização em cinco fases principais: coagulação, inflamação, proliferação, contração da ferida e remodelação.

Sempre houve a preocupação de se tentar neoformação do tecido traumatizado morfológica e fisiologicamente semelhantes ao tecido perdido e, diante disto, artifícios que favoreçam o processo de cicatrização são utilizados, como o uso de fitoterápicos e laser. A utilização de plantas já consta em registros egípcios antigos ${ }^{7}$ com a finalidade de buscar princípios ativos que desempenhem efetivo papel no processo de cicatrização acelerando desta maneira a recuperação cirúrgica. Entre estas substâncias pode-se citar a Pfaffia glomerata (Spreng ) Pedersen, Amaranthaceae, que é uma das espécies do ginseng brasileiro usada como planta medicinal ${ }^{8}$. Ela recebe este nome no Brasil devido ao formato de suas raízes serem muito semelhantes às do ginseng coreano (Panax ginseng).

A Pfaffia glomerata é utilizada na medicina popular como tônico, antidiabético, para melhorar o processo de cicatrização, assim como para neutralizar distúrbios gástrico e antirreumático ${ }^{4}$. Outras propriedades são a redução da inflamação, o alívio da dor, o aumento da imunidade e a oxigenação de células, além de baixo custo.

Já o laser de baixa potência tem despertado interesse nos pesquisadores, uma vez que devido à existência de células que possuem elementos que reagem à luz (cromóforos), a bioestimulação poderia ser alcançada através do uso deste recurso.

$\mathrm{O}$ uso do laser de baixa potência em tecidos moles tem mostrado que o efeito anti-inflamatório, a redução da dor e a aceleração da proliferação celular - em resumo a melhora no processo de cicatrização - depende do comprimento de onda, dose e condição local ${ }^{5}$. O laser de baixa potência tem a capacidade de penetrar nos tecidos, atuando na síntese do colágeno, no estímulo da circulação local e aumentando a atividade mitótica das células epiteliais. No entretanto, é aparelho de alto custo.

O objetivo deste trabalho é comparar a contração da ferida e a neovascularização com o uso da Pfaffia glomerata e do laser de baixa potência em dorso de ratos.

\section{MÉTODOS}

Este estudo foi realizado no Instituto de Pesquisas Médicas (IPEM) do Programa de Pós-graduação em Princípios da Cirurgia do Hospital Universitário Evangélico de Curitiba e Faculdade Evangélica do Paraná (FEPAR) e as análises histológicas no laboratório Citolab. Ele foi aprovado pelo Comitê de Ética em Pesquisa da Sociedade Evangélica Beneficente de Curitiba.

Obtenção do extrato da planta pfaffia glomerata Esta etapa foi realizada no Laboratório de Farmacognosia da Universidade Federal do Paraná, Curitiba, PR, Brasil. A planta já processada em estado sólido (pó) foi adquirida da E. Martins Produtos Naturais.

Em um percolador foram acondicionados as raízes trituradas da planta e solução de etanol a 50\% em água deionizada e deixados em maceração por três dias. Após esse período, o extrato resultante foi filtrado à vácuo e concentrado em rotavapor com temperatura abaixo de 50 (Figuras 1 e 2). $O$ extrato total foi conservado em geladeira à $4^{\circ} \mathrm{C}$ até homogenização com propilenoglicol, veículo para facilitar a administração. A concentração da Pfaffia no extrato final foi de $23,90 \%$. A droga obtida foi conservada novamente em geladeira à $4{ }^{\circ} \mathrm{C}$

Após a obtenção do extrato, seguiu-se a realização de etapa experimental em cinco ratos para avaliação da toxicidade da concentração obtida.

\section{Caracterização da amostra}

Foram utilizados 80 ratos (Rattus novergicus albinus, Rodentia mammalia), da linhagem Wistar, machos, procedentes do biotério do Instituto de Tecnologia do Paraná com idade de 120 dias e peso médio de $230 \mathrm{~g}$.

Em 40 animais foram realizadas duas feridas cirúrgicas para caracterizar o grupo controle, no 


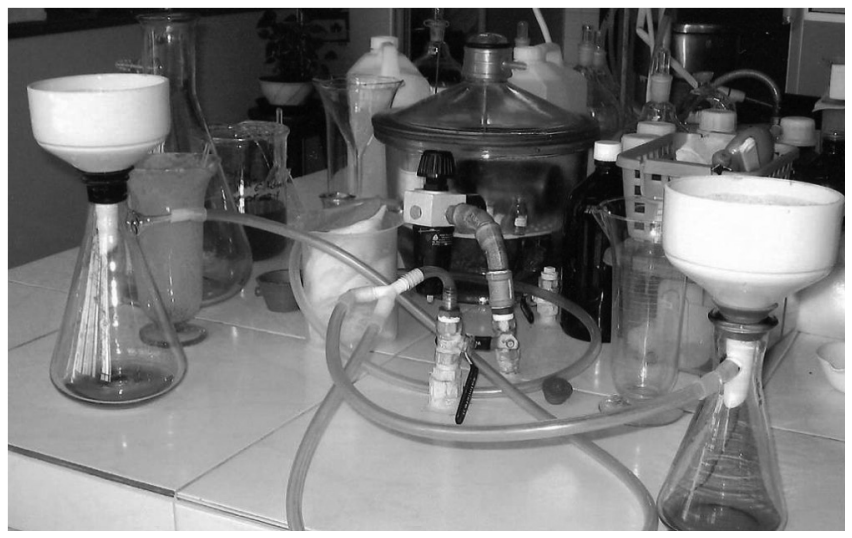

FIGURA 1 - Extrato sendo filtrado a vácuo

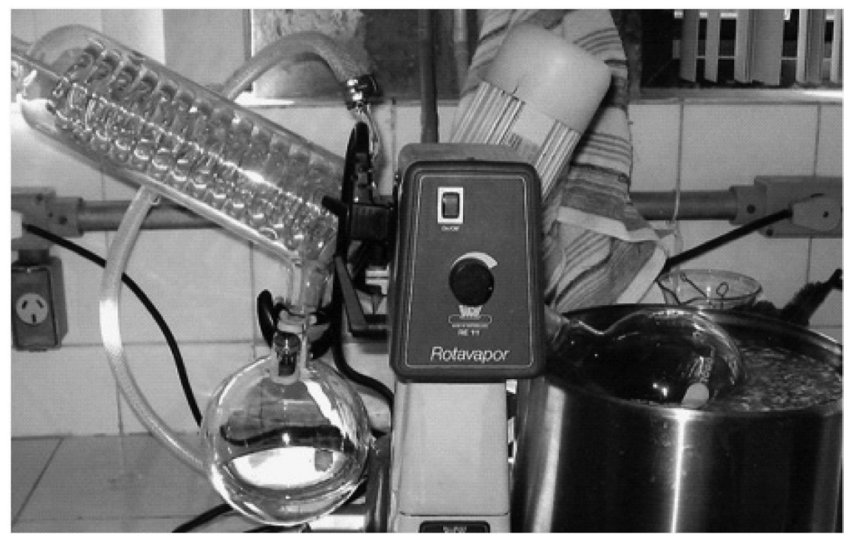

FIGURA 2 - Rotavapor utilizado para a evaporação do etanol

dorso da parte superior onde nenhuma substância foi utilizada, e na região inferior foi aplicado com um conta-gotas o extrato de Pfaffia glomerata para que o mesmo não escoasse para as feridas cirúrgicas de controle e alterasse, desta maneira o resultado deste grupo. Assim sendo, esses dois grupos foram realizados nos mesmos animais.

Nos outros 40 ratos também foram realizadas feridas cirúrgicas com o mesmo instrumento, para a padronização das mesmas, só que nestes animais foi realizada uma única ferida para a aplicação do laser de baixa potência de Hélio-Neônio, infravermelho, com comprimento de onda de $785 \mathrm{~nm}$, da marca Kondortech ${ }^{\circledR}$, modelo DL 7140-211S, com irradiação de $2 \mathrm{~J} / \mathrm{cm}^{2}$, durante o tempo de 50 segundos.

Assim sendo a pesquisa constituiu-se de três grupos: controle, planta e laser e quatro sub-grupos, conforme o dia do sacrifício, em 48 horas, uma, duas e três semanas

Os animais foram pesados e submetidos à anestesia geral com administração intramuscular de Ketamina (Ketalar ${ }^{\circledR}$ Aché) na dose de $50 \mathrm{mg} / \mathrm{Kg}$ e xilazina (Virbaxyl 2\% ${ }^{\circledR}$ Virbac) na dose de $10 \mathrm{mg} /$ Kg. Após a aplicação da medicação aguardou-se até que o animal permanecesse inconsciente e imóvel ao manuseio, considerando-o assim como anestesiado. Os ratos permaneceram em plano anestésico profundo até o final da operação.
A seguir, cada animal foi posicionado em decúbito ventral, sem o uso de imobilização, para a realização da tricotomia na região dorsal, feita com lâmina e sabão líquido neutro. Após a realização de um template em folha de transparência com $8 \mathrm{~mm}$ de largura e no seu centro uma circunferência de $3 \mathrm{~mm}$ que coincidia com o tamanho da lesão, que foi marcada com caneta de tinta solúvel em álcool. Nos animais que serviram de controle e experimento (planta) os pontos de referência eram o ângulo inferior da escápula para o grupo controle e a borda superior do osso ilíaco para o grupo planta. Já para os animais onde foi aplicado o laser, a referência era a linha média dorsal. Procedeu-se a antissepsia com solução alcólica de clorexidine $2 \%$ antes da realização da ferida cirúrgica, sendo que ela foi executada com punch de $3 \mathrm{~mm}$ de diâmetro.

Após a operação os animais foram acondicionados em gaiolas, contendo cinco em cada, mantidos no biotério do IPEM, em temperatura ambiente, com ciclo claro-escuro e com livre acesso à água potável e ração padrão comercial (Nuvilab ${ }^{\circledR}$ Nuvital). Todas as gaiolas foram limpas, e a ração e água trocadas diariamente. Eles não receberam curativos no pós-operatório e permaneceram sob este regime até a data prevista para as aferições do experimento.

Conforme cronograma estabelecido, os ratos foram mortos utilizando a solução do anestésico barbitúrico Sodium Thiopental ${ }^{\circledR}$, na concentração de $1 \mathrm{~g}$ diluído em $40 \mathrm{~mL}$ de soro fisiológico, injetada no peritônio. Depois de constatada a morte verificada através da midríase pupilar, o rato era posicionado em decúbito ventral, sem imobilização e realizada a aferição da medida do diâmetro da ferida com uma régua metálica graduada em centímetros. Em seguida, com uma lâmina de bisturi, incisou-se a pele na demarcação anteriormente feita e a peça obtida era depositada em frascos contendo solução de formol à 10\% para então ser encaminhada para a análise histológica.

\section{Avaliações macro e microscópicas}

Em cada dia da morte dos animais eram tomadas as medidas no centro para analisar a contração da ferida e anotadas em tabela.

Na microscopia, o material foi fixado em solução de formol à $10 \%$. Procedeu-se a técnica histológica padrão para hematoxilina e eosina $(\mathrm{HE})$, na intenção de verificação do processo inflamatório. Através da imunoistoquímica com Fator VIII verificou-se a densidade vascular, sendo a marcação revelada com o cromógeno diaminobenzidina, dando coloração marrom à marcação positiva do citado anticorpo. A observação da fibrose foi realizada através da coloração de tricrômio de Masson (com azul de anilina), sendo a análise realizada na área mais significativa com relação à presença das fibras colágenas.

Os dados foram organizados em planilha Excel e analisados através do programa computacional Statistica, versão 8.0. Quando da comparação dos grupos 
Controle e Planta, adotou-se o teste não paramétrico de Wilcoxon em função do pareamento. Nas comparações dos grupos Planta e Controle com o grupo Laser foi considerado o teste não paramétrico de Mann-Whitney em função de serem grupos independentes. Valores de $p<0,05$ indicaram significância estatística.

\section{RESULTADOS}

Não houve diferença estatística entre os subgrupos no grupo controle. Em relação ao grupo planta a menor média ocorreu no sub-grupo de três semanas, porém a diferença não foi estatisticamente significante (Tabela 1).

TABELA 1 - Comparação dos subgrupos dentro do grupo planta

\begin{tabular}{|c|c|c|c|c|c|c|}
\hline Sub-grupo & $\mathrm{n}$ & média & mediana & mínimo & máximo & $\begin{array}{l}\text { desvio- } \\
\text { padrão }\end{array}$ \\
\hline 48 horas & 10 & 2,7 & 2,75 & 2 & 3 & 0,3 \\
\hline 1 sem. & 10 & 2,4 & 2,25 & 2 & 3 & 0,4 \\
\hline 2 sem. & 10 & 2,2 & 2 & 2 & 3 & 0,4 \\
\hline 3 sem. & 10 & 1,8 & 2 & 1 & 2 & 0,4 \\
\hline & & & & & & $\mathrm{p}$ \\
\hline \multicolumn{6}{|c|}{48 horas $x 1$ semana } & 0,050 \\
\hline \multicolumn{6}{|c|}{1 semana $\times 2$ semanas } & 0,418 \\
\hline \multicolumn{6}{|c|}{2 semanas $\times 3$ semanas } & 0,109 \\
\hline
\end{tabular}

Em relação ao grupo laser a menor média para o grupo laser ocorreu no tempo de três semanas com diferença estatisticamente significante na comparação dos sub-grupos 48 horas versus uma semana (Tabela 2 ).

TABELA 2 - Comparação dos sub-grupos dentro do grupo laser

\begin{tabular}{|c|c|c|c|c|c|c|}
\hline Sub-grupo & $\mathrm{n}$ & média & mediana & mínimo & máximo & $\begin{array}{l}\text { desvio- } \\
\text { padrão }\end{array}$ \\
\hline 48 horas & 10 & 2,8 & 3 & 2,5 & 3 & 0,3 \\
\hline $1 \mathrm{sem}$. & 10 & 2,4 & 2,5 & 2 & 3 & 0,3 \\
\hline 2 sem. & 10 & 2,1 & 2 & 2 & 3 & 0,3 \\
\hline 3 sem. & 10 & 1,8 & 2 & 1 & 2 & 0,4 \\
\hline & & & & & & $p$ \\
\hline \multicolumn{6}{|c|}{48 horas $\times 1$ semana } & 0,008 \\
\hline \multicolumn{6}{|c|}{1 semana $\times 2$ semanas } & 0,245 \\
\hline \multicolumn{6}{|c|}{2 semanas $\times 3$ semanas } & 0,109 \\
\hline
\end{tabular}

\section{Comparação entre os grupos}

A menor média do grupo controle foi no tempo de três semanas, cujo valor se apresentou de 2,0; no grupo planta, o menor valor da média foi de 1,8 neste mesmo tempo e quanto ao grupo laser, a menor média também se apresentou com 0 valor de 1,8 , neste mesmo sub-grupo (Figura 3). Assim sendo, os grupos planta e laser apresentaram a mesma contração da ferida no final do experimento. Porém somente o grupo laser teve valor de $p$ significante na comparação dos sub-grupos 48 horas e uma semana $(p=0,008)$, pois a média em 48 horas foi de 2,8 e de uma semana de 2,4 , indicando maior contração da ferida durante esses tempos.

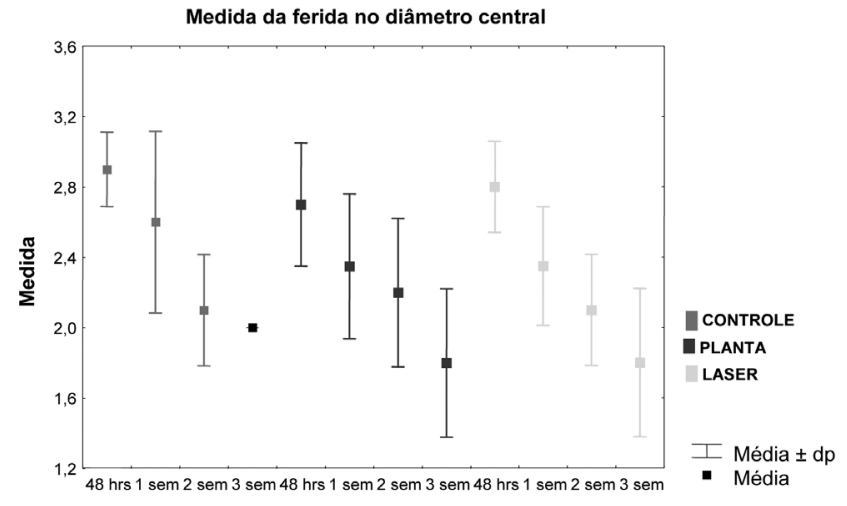

FIGURA 3 - Representação da contração da ferida nos subgrupos dentro de cada grupo

\section{Avaliação microscópica}

$\mathrm{Na}$ avaliação imunoistoquímica com Fator VIII no grupo controle a diferença estatisticamente significante ocorreu quando comparados os sub-grupos 48 horas com uma semana $(p=0,011)$ e uma semana com duas semanas $(p=0,035)$.

No grupo planta a diferença estatisticamente significante ocorreu na comparação dos sub-grupos uma semana com duas semanas $(p=0,001)$ (Tabela 3$)$

TABELA 3 - Comparação dos subgrupos dentro do grupo planta

\begin{tabular}{|c|c|c|c|c|c|c|}
\hline Sub-grupo & $\mathrm{n}$ & média & mediana & mínimo & máximo & $\begin{array}{l}\text { desvio- } \\
\text { padrão }\end{array}$ \\
\hline 48 horas & 10 & 11,8 & 13 & 8 & 14 & 2 \\
\hline $1 \mathrm{sem}$. & 10 & 14,1 & 13 & 11 & 19 & 3,11 \\
\hline 2 sem. & 10 & 9,4 & 9 & 7 & 13 & 1,78 \\
\hline 3 sem. & 10 & 9 & 9 & 7 & 15 & 2,39 \\
\hline & 3 & & & & & $p$ \\
\hline \multicolumn{6}{|c|}{48 horas $x 1$ semana } & 0,247 \\
\hline \multicolumn{6}{|c|}{1 semana $\times 2$ semanas } & $<0,001$ \\
\hline \multicolumn{6}{|c|}{2 semanas $\times 3$ semanas } & 0,436 \\
\hline
\end{tabular}

No grupo laser, a diferença estatisticamente significante ocorreu na comparação dos sub-grupos uma semana com duas semanas $(p=0,001)$ (Tabela 4).

TABELA 4 - Comparação dos sub-grupos dentro do grupo laser

\begin{tabular}{|c|c|c|c|c|c|c|}
\hline Sub-grupo & $\mathrm{n}$ & média & mediana & mínimo & máximo & $\begin{array}{l}\text { desvio- } \\
\text { padrão }\end{array}$ \\
\hline 48 horas & 10 & 10,5 & 10,8 & 7 & 13 & 2,17 \\
\hline $1 \mathrm{sem}$. & 10 & 11 & 11 & 9 & 17 & 2,49 \\
\hline 2 sem. & 10 & 8,1 & 8 & 7 & 10 & 0,99 \\
\hline 3 sem. & 10 & 8,5 & 8 & 6,5 & 13 & 1,89 \\
\hline & $p$ & & & & & $p$ \\
\hline \multicolumn{6}{|c|}{48 horas $\times 1$ semana } & 0,739 \\
\hline \multicolumn{6}{|c|}{1 semana $\times 2$ semanas } & $<0,001$ \\
\hline \multicolumn{6}{|c|}{2 semanas $\times 3$ semanas } & 0,971 \\
\hline
\end{tabular}

A comparação dos grupos dentro dos sub-grupos, o grupo controle teve maior média no tempo de uma semana (valor=12,9). O grupo planta, apresentou também sua maior média no tempo uma semana com o valor de 
14,1 e o grupo laser, também neste tempo, apresentou média no valor de 11,0 . Através destas médias, o valor de $\mathrm{p}$ obtido foi significante na comparação entre planta e laser neste tempo de uma semana com o valor de 0,009 . Sendo assim, neste sub-grupo os resultados foram superiores para o grupo planta. Isto significa que a diferença estatisticamente significante ocorreu neste sub-grupo para este grupo e que após este tempo houve equilíbrio nos resultados obtidos.

\section{DISCUSSÃO}

A pele do rato apresenta diferença importante em relação à humana, que é a ausência de um limite definido entre derme papilar e reticular, o que pode falsear resultados de trabalhos experimentais ${ }^{1,2,3}$. Contudo há referência ao contrário ${ }^{9}$, referindo que a deposição de fibras colágenas em ratos - por serem também animais mamíferos -, tem os componentes do processo de cicatrização semelhantes ao humano, o que permite a comparação dos resultados obtidos, embora o acelerado metabolismo do rato percorra mais rapidamente as fases inflamatória, de formação tecidual e de remodelação.

Autores $^{1}$ relatam que o porco é o animal que tem sido mais utilizado em estudos experimentais com lasers, pois sua pele apresenta grande analogia com a estrutura e cicatrização da pele humana. Além disso, a pele dele se assemelha à humana também quanto ao número de folículos pilosos e processo de epitelização. Entretanto, as dificuldades decorrentes de sua obtenção, manipulação, manutenção e ainda o grande porte destes animais são obstáculos reais para sua utilização em estudos experimentais.

Quanto ao local da realização do procedimento cirúrgico, o dorso do animal é preferencial, pois desta maneira não há condições dele coçar as feridas, causando alteração dos resultados e nem retirar as demarcações feitas, lambendo-as ${ }^{1}$.

A avaliação macroscópica que constou da verificação da contração da ferida tendo como referência as medidas demarcadas no dia do procedimento cirúrgico, mostrou-se adequada ${ }^{1}$ embora estudo tensiométrico possa ser também utilizado9. Essa verificação se fez necessária, uma vez que o processo de contração da ferida é a quarta fase da cicatrização e consiste no movimento centrípeto das bordas ${ }^{7}$.

A avaliação microscópica foi analisada através da imunoistoquímica, para a verificação da neovascularização - Fator VIII - que é uma glicoproteína produzida unicamente pelas células endoteliais e megacariócitos. Por este motivo, é rotineiramente utilizada para identificar vasos em cortes de tecidos. Isto está de acordo com Xavier ${ }^{10}$, que relata ser ele um anticorpo monoclonal capaz de reagir com células endoteliais, exibindo reatividade em padrão granular ao nível do citoplasma das células marcadas positivamente. Neste contexto, e tomando como referência Mandelbaum, et al. ${ }^{7}$ quando relatam que a última subfase da proliferação é a angiogênese essencial para o suprimento de oxigênio e nutrientes para a cicatrização, obteve-se melhor resultado com o grupo planta no período de uma semana.

\section{CONCLUSÕES}

Não houve diferença estatisticamente significante com relação à contração da ferida entre os grupos planta e laser, obtendo ambos resultados superiores ao grupo controle no final do estudo. Contudo, microscopicamente, o grupo planta obteve valor superior ao grupo laser quanto à neovascularização em uma semana; após esse período, houve equilíbrio com os outros grupos.

\section{REFERÊNCIAS}

1. Araújo, LRR. Estudo comparativo dos efeitos induzidos pela aplicação do laser de $\mathrm{CO} 2$ ultrapussado e do laser de Erbium: Yttrium Aluminum Garnet, em pele de ratos. [Dissertação] Programa de Pós-Graduação em Princípios da Cirurgia da Faculdade Evangélica do Paraná / Hospital Universitário Evangélico de Curitiba, 2001.

2. Bittencourt LM. Avaliação das alterações histológicas e da aderência decorrentes da colocação de implantes de silicone revestido com espuma de poliuretano e com espuma de silicone no dorso de ratos. [Dissertação] Programa de Pós-Graduação em Princípios da Cirurgia do Instituto de Pesquisas Médicas da Faculdade Evangélica do Paraná, Curitiba, 2008.

3. Coelho JC, Antoniolli AB, Silva DN, Carvalho TMMB, Pontes ERJC, Odashiro AN. O efeito da sulfadiazina de prata, extrato de ipê-roxo e extrato de barbatimão na cicatrização de feridas cutâneas em ratos. Rev Col Bras Cir. 2010;37(1):45-51.

4. Freitas CS, Baggio $\mathrm{CH}$, Silva-Santos JE, Rieck L, Santos CAM, Júnior CC, Ming LC, Cortez DAG, Marques MCA. Involvement of nitric oxide in the gastroprotective effects of na aqueous extract of Pfaffia glomerata ( Spreng. ) Pedersen, Amaranthaceae, in rats. Life Sciences 2004; 74:1167-79.

5. Júnior ANS, Pinheiro ALB, Oliveira MG, Weismann R, Ramalho LMP, Nicolau RA. Computadorized morphometric assessment of the effect of low-level laser therapy on bone repair: na experimental animal study. J. Clin. Laser Med. \& Surg 2002;20(2):83-7.

6. Júnior JB. Laser de baixa potência no reparo de feridas em mucosa bucal de coelhos (Oryctolagos Cuniculus). [Tese] Programa de Pós-Graduação em Clínica Cirúrgica, Setor de Ciências da Saúde, Universidade Federal do Paraná, Curitiba 2005.

7. Mandelbaum SH, Di Santis EP, Mandelbaum MHS. Cicatrização: conceitos atuais e recursos auxiliares - Parte I. An bras Dermatol. 2003;78(4):393-410.

8. Nicoloso FT, Erig AC, Martins CF, Russowski D. Micropropagação do ginseng brasileiro [Pfaffia glomerata (Spreng.) Pedersen]. Rev Bras PL Med 2001;3(2):11-8.

9. Rodrigues RG. Efeitos do laser de CO2 ultrapulsado na viabilidade, contração e fibroplasia de retalho cutâneo dorsal de ratos. [Tese] Programa de Pós-Graduação em Clínica Cirúrgica do Setor de Ciências da Saúde da Universidade Federal do Paraná, Curitiba, 2001.

10. Xavier RLF. Estudo da angiogênese pelo CD 105 e FvW no carcinoma epidermóide oral e sua relação com o estadiamento clínico do tumor. [Dissertação] Programa de Pós -Graduação em Patologia Oral/ Universidade Federal do Rio Grande do Norte/ Centro de Ciências da Saúde/ Departamento de Odontologia, Natal, 2008. 